\title{
CDK inhibitor enhances the sensitivity to 5-fluorouracil in colorectal cancer cells
}

\author{
KOICHI TAKAGI ${ }^{1}$, YOSHIHIRO SOWA ${ }^{1}$, OZGUR MUHAMMER CEVIK ${ }^{1,2}$, \\ RYOKO NAKANISHI $^{1}$ and TOSHIYUKI SAKAI ${ }^{1}$ \\ Departments of ${ }^{1}$ Molecular-Targeting Cancer Prevention, ${ }^{2}$ Pharmacology, Graduate School \\ of Medical Science, Kyoto Prefectural University of Medicine, Kyoto, Japan
}

Received December 31, 2007; Accepted February 22, 2008

\begin{abstract}
Thymidylate synthase (TS) is a dNTP synthetic enzyme and is also a target enzyme of 5-fluorouracil (5-FU). 5 -FU is one of the anticancer agents most frequently used for the treatment of colorectal cancers. However, the clinical rate of response to its use as a single agent is not exceptionally high. Therefore, various combination chemotherapies have been devised. The elevated expression of TS in cancer cells is a serious obstacle in the clinical use of 5-FU. In the present study, TS expression was up-regulated by the knockout of the p21 $1^{\text {WAFI/CIPI }}$ gene in human colorectal cancer HCT116 cells, suggesting that TS expression is mediated through the inhibition of cyclin-dependent kinase (CDK). Based on these findings, we tested whether the CDK inhibitor (CDKI) SU9516, acted as a suppressor of TS. SU9516 effectively reduced the expression of TS in a dose-dependent manner. Furthermore, the reduction of TS expression resulted in enhancement of the sensitivity to 5-FU in human colon cancer DLD-1 cells. Thus, SU9516 might be a promising compound for combination chemotherapy with 5-FU.
\end{abstract}

\section{Introduction}

Colorectal cancers often show low sensitivity to anticancer agents. 5-Fluorouracil (5-FU) has been in clinical use as an anticancer agent and remains a mainstay of chemotherapeutic approaches for the treatment of colorectal cancers (1). However, the clinical rate of response to its use as a single

Correspondence to: Dr Yoshihiro Sowa, Department of MolecularTargeting Cancer Prevention, Graduate School of Medical Science, Kyoto Prefectural University of Medicine, Kawaramachi-Hirokoji, Kamigyo-ku, Kyoto 602-8566, Japan

E-mail: ysowa@koto.kpu-m.ac.jp

Abbreviations: TS, thymidylate synthase; GAPDH, glyceraldehyde3-phosphate dehydrogenase

Key words: colorectal cancer, 5-fluorouracil, retinoblastoma tumor suppressor, thymidylate synthase, cyclin-dependent kinase inhibitor agent is not exceptionally high. Therefore, various combination chemotherapies have been devised.

The target enzyme of 5-FU is thymidylate synthase (TS). TS is a folate-dependent enzyme that is essential for DNA synthesis and DNA repair. TS often functions as an oncogene (2) and inhibition of the TS reaction results in the cessation of cellular proliferation and growth. Thus, TS represents an important target for cancer chemotherapy. However, the expression levels of TS are elevated in many cases, which is a serious obstacle in the clinical use of 5-FU (3). Accordingly, clinical studies have shown that high TS levels are correlated with poor prognosis in patients with colorectal and other malignant tumors (4). To make matters worse, exposure to TS inhibitors leads to the further up-regulation of TS expression (5). Therefore, some experiments for regulating TS expression have been attempted, including TS-targeted siRNAs that may have therapeutic potential by themselves or as chemosensitizers in combination with $5-\mathrm{FU}(6,7)$.

On the other hand, the expression of TS has been assumed to be up-regulated by E2F1 (8). Furthermore, it has been reported that the retinoblastoma tumor-suppressor (RB) protein which acts as a negative regulator of cell cycle transition, suppresses the expression of TS $(9,10)$. RB is also known to bind the E2F family of transcriptional factors. In quiescent cells, the function of E2F is inactivated by binding to the unphosphorylated form of RB protein (11). When RB is phosphorylated by CDK-cyclins, E2F is released from the complex and binds to a cis-element in the promoter region of various genes involved in cell proliferation and DNA synthesis, such as TS (8). Thus, several studies have shown that TS expression is correlated with E2F1 expression $(12,13)$.

Based on these findings, we investigated whether a CDK inhibitor (CDKI) acted as a suppressor of TS, and whether it could be used to enhance the sensitivity to 5-FU. Our results demonstrated that SU9516, a CDKI $(14,15)$ effectively reduced TS expression and enhanced the sensitivity to 5-FU in human colorectal cancer cells.

\section{Materials and methods}

Reagents. FdUrd, a metabolized product of 5-FU, was obtained from Wako (Osaka, Japan). SU9516 was obtained from Calbiochem (San Diego, CA). They were dissolved in 
dimethylsulfoxide (DMSO) and diluted to the final concentrations in each culture medium used.

Cell culture. Human colon adenocarcinoma DLD-1 cells and FdUrd-resistant DLD-1 cells (DLD-1/FdUrd cells), a kind gift from Taiho Pharmaceutical Co. Ltd. (Tokushima, Japan), were cultured in Roswell Park Memorial Institute (RPMI) medium. The establishment of DLD-1/FdUrd cells was described previously (16). Human colon adenocarcinoma wild-type HCT116 cells (HCT116 WT cells) and p21 WAFI/CIPI null HCT116 cells (HCT116 p21\% cells), a kind gift from Dr B. Vogelstein (The Johns Hopkins University, Baltimore, MD), were cultured in Dulbecco's modified Eagle's medium (DMEM). The establishment of HCT116 p $21^{-/}$cells was described previously (17). Each medium contained $10 \%$ fetal bovine serum and $2 \mathrm{mM}$ glutamine (RPMI) or $4 \mathrm{mM}$ glutamine (DMEM). All cells were maintained at $37^{\circ} \mathrm{C}$ in a humidified atmosphere supplemented with $5 \% \mathrm{CO}_{2}$.

Colony formation assay. For the cell growth study, DLD-1, DLD-1/FdUrd, HCT116 WT and p21/- cells were seeded at a density of 200 cells in each well of 6-well plates. After culturing for $24 \mathrm{~h}$, cells were treated with various concentrations of FdUrd for $48 \mathrm{~h}$. Then the cells were incubated in growth medium for 7-10 days, and the number of viable colonies was counted. For the study of the effect of SU9516, DLD-1 cells were seeded at a density of 200 cells in each well of 6-well plates. After culturing for $24 \mathrm{~h}$, cells were exposed to SU9516 $(5 \mu \mathrm{M})$ or mock-treated with DMSO, and $24 \mathrm{~h}$ after the treatment, growth medium was replaced by medium supplemented with various concentrations of FdUrd and culturing was continued for $48 \mathrm{~h}$. In the same way as in the cell growth study, cells were incubated in growth medium for 7-10 days, and the number of viable colonies was counted. The data are presented as the percent compared to the control samples and were analyzed using the Student's t-test. Differences were considered to be statistically significant from the controls at $\mathrm{p}<0.05$.

Western blot analysis. Cells were lysed with buffer containing $50 \mathrm{mM}$ Tris- $\mathrm{HCl}$ (pH 6.8), 10\% glycerol, 1\% SDS, 5\% 2 -mercaptoethanol and $0.025 \%$ bromphenol blue. The protein extract was loaded onto a $12 \%$ (for TS and GAPDH detection) or $7 \%$ (for RB detection) polyacrylamide gel, electrophoresed, and transferred onto a polyvinylidene difluoride membrane (Millipore, Bedford, MA). Monoclonal antibodies to TS [a kind gift from Taiho Pharmaceutical Co. Ltd. (18)], RB (PM-14001A from Pharmingen, Franklin Lakes, NJ), phsphorylated RB (Thr356) (Biosource, Carlsbad, CA) or GAPDH (HyTest Ltd., Turku, Finland) were used as the primary antibodies. The signal was then developed with Chemi-Lumi One (Nacalai Tesque Inc., Kyoto, Japan).

RNA isolation and quantitative real-time RT-PCR. Total RNA was isolated from DLD-1 cells using Sepasol-RNA I (Nacalai Tesque Inc.). Total RNA $(10 \mu \mathrm{g})$ was reverse transcribed to cDNA in a $20-\mu 1$ reaction volume, with Superscript reverse transcriptase (Invitrogen Corp., Carlsbad, CA), using oligo(dT) primers (Toyobo Co., Ltd., Osaka, Japan). The reaction mixture was incubated at $42^{\circ} \mathrm{C}$ for $50 \mathrm{~min}$, and then at $70^{\circ} \mathrm{C}$ for $15 \mathrm{~min}$ to stop the reaction. An equivalent volume $(1 \mu \mathrm{l})$ of cDNA solution was used for the quantification of specific cDNAs by quantitative real-time RT-PCR. The primer sequences used for the TS and GAPDH genes were described previously (19). Quantitative real-time RT-PCR was carried out using an RT-PCR system GeneAmp5700 (Applied Biosystems, Foster, CA). The expression level of TS mRNA was normalized by the level of GAPDH mRNA in the same sample.

DNA transfection and luciferase assay. The TS promoter plasmid was a kind gift from Dr K. Kawakami (20). For the establishment of a TS mutant promoter, a QuickChange sitedirected mutagenesis kit (Stratagene Cloning Systems, Co., La Jolla, CA) was used to generate point mutations at the E2F site of the TS reporter plasmid. The mutant sense sequence was CGGGACGGCCGCtaGAAAAGGCGCG CGG (the point mutation is shown as a small letter) and the wild-type sense sequence was CGGGACGGCCGCGGGAAA AGGCGCGCGG (the consensus E2F sequence site in the TS promoter between -129 and $-156 \mathrm{bp}$ from the first nucleotide of the initiation codon is underlined). DLD- 1 cells $\left(1 \times 10^{4}\right.$ cells $)$ were incubated in each well of 12-well plates, and $1.0 \mu \mathrm{g}$ per well of a reporter plasmid DNA was transfected using Lipofectamine Plus reagent (Invitrogen Corp.). For the study of the effect of $\mathrm{p} 21^{\text {WAFI/CIPI }}$ or E2F1 expression on the TS promoter, $1.0 \mu \mathrm{g}$ per well of each expression vector was transfected at the same time. After $24 \mathrm{~h}$, the cells were harvested. For the study of the effect of SU9516, $24 \mathrm{~h}$ after transfection of the TS promoter, the cells were treated with medium containing SU9516, and $24 \mathrm{~h}$ after the start of the treatment, the cells were harvested for the luciferase assay. Luciferase activity was analyzed using a PicaGene luminescence kit (Toyo Ink, Tokyo, Japan) and a luminometer. The relative luciferase activity was standardized by the protein concentration of each cell lysate.

\section{Results}

The expression levels of TS and the sensitivity to FdUrd in DLD-1 and DLD-1/FdUrd cells. We examined the expression levels of TS in DLD-1 and DLD-1/FdUrd cells by Western blotting. TS expression was higher in DLD-1/FdUrd than in DLD-1 cells (Fig. 1A). We subsequently confirmed the sensitivity of DLD-1 and DLD-1/FdUrd cells to FdUrd in vitro by colony formation assays. As shown in Fig. 1B, upon exposure to various concentrations of FdUrd for $48 \mathrm{~h}$, DLD-1/FdUrd cells showed a smaller reduction in the colony number than DLD-1 cells in accord with a previous study (21), indicating that TS expression is reversely correlated with the sensitivity to FdUrd. These findings also agree with a previous study showing that further up-regulation of TS expression by exposure to TS inhibitors leads to a more critical condition (5).

The expression levels of TS and the sensitivity to FdUrd in HCT116 WT and p21 $1^{-/-}$cells. To test whether CDKI acted as a suppressor of TS, we used HCT116 WT and p21/- cells, because p21 acts as a CDK inhibitor. After confirming that the expression of p21 was not detected in HCT116 p $21^{-/-}$ 
A

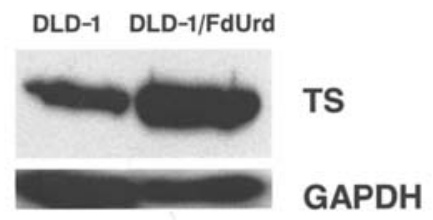

B

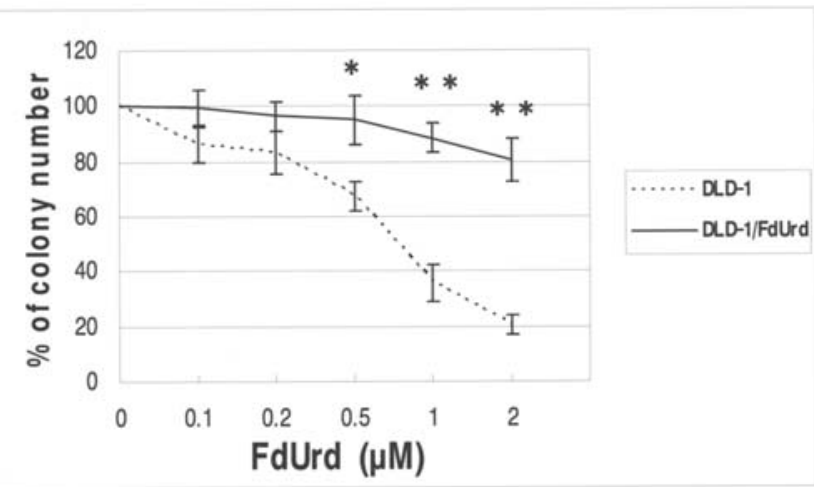

Figure 1. (A) The levels of TS protein of DLD-1 and DLD-1/FdUrd cells. GAPDH was used as an internal control. (B) Sensitivity of DLD-1 and DLD-1/ FdUrd cells to FdUrd. Cells were exposed to various concentrations of FdUrd for $48 \mathrm{~h}$, and the number of viable colonies was counted $\left({ }^{*} \mathrm{p}<0.05 ;{ }^{* *} \mathrm{p}<0.01\right)$.

A

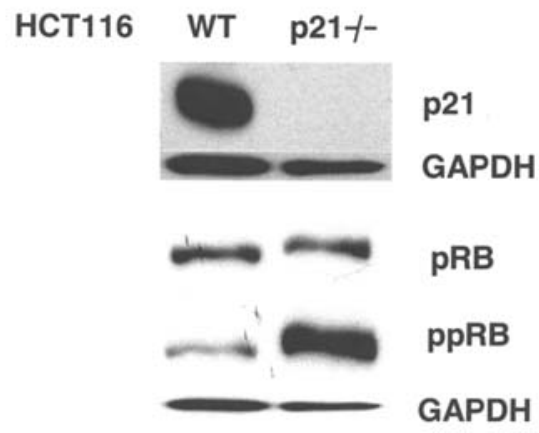

C

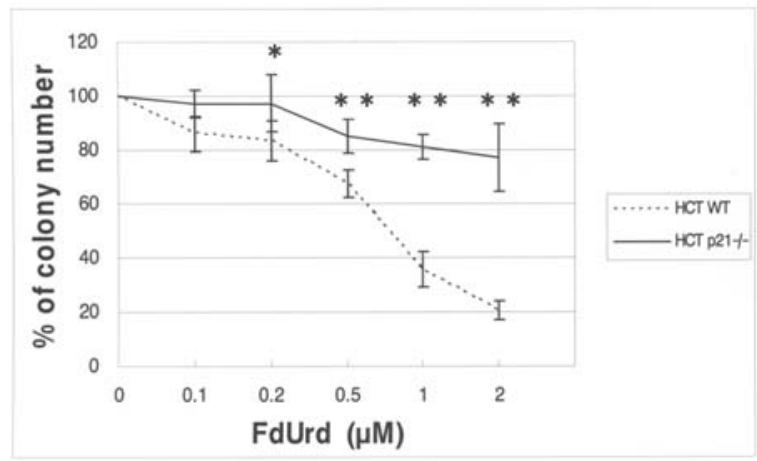

cells, we examined phosphorylated RB in HCT116 WT and $\mathrm{p} 21^{-/}$cells by Western blotting. The expression of phosphorylated RB was higher in p21/- than in WT cells (Fig. 2A). Then, the expression levels of TS in HCT116 WT and p21 ${ }^{-1}$ cells were examined by Western blotting. As shown in Fig. 2B, TS expression was higher in HCT116 $\mathrm{p} 21^{-/-}$than in WT cells. We were able to assess the relationship between $\mathrm{p} 21$ and TS expression. Subsequently, we examined the sensitivity of HCT116 WT and p21/- cells to FdUrd in vitro by a colony formation assay. Upon exposure to various concentrations of FdUrd for $48 \mathrm{~h}$, HCT116 p $21^{/-}$cells showed a smaller reduction in the colony number than WT cells, indicating CDKI affects the sensitivity to FdUrd (Fig. 2C).
B

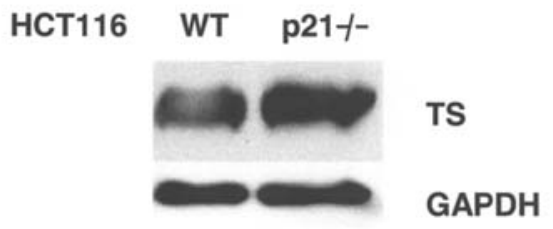

Figure 2. (A) The levels of p21 and phosphorylated RB of HCT116 WT and p $21^{-/}$cells. GAPDH was used as an internal control. (B) The levels of TS protein of HCT116 WT and p $21^{-/}$cells. GAPDH was used as anternal control. (C) Sensitivity of HCT116 WT and p21\% cells to FdUrd. Cells were exposed to various concentrations of FdUrd for $48 \mathrm{~h}$, and the number of viable colonies was counted $\left({ }^{*} \mathrm{p}<0.05 ;{ }^{* *} \mathrm{p}<0.01\right)$.

Effects of ectopic p21 and E2F expression on TS promoter activity. To ascertain whether the down-regulation of the TS gene expression is mediated through the CDK-RB pathway, we employed p21 and E2F expression vectors, and studied the effects on the TS promoter. As shown in Fig. 3A, the TS promoter activity was down-regulated by ectopic p 21 expression. On the other hand, the TS promoter activity was up-regulated by ectopic E2F expression (Fig. 3B). Furthermore, to determine whether a region of the TS promoter is responsible for the activation by E2F, we generated point mutations at the E2F site of the TS promoter. As shown in Fig. 3C, the TS mutant promoter activity was not up-regulated by ectopic E2F expression. 
A

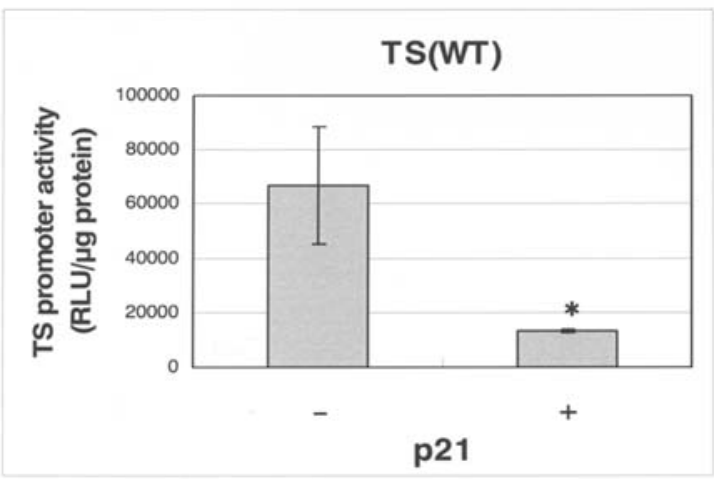

B

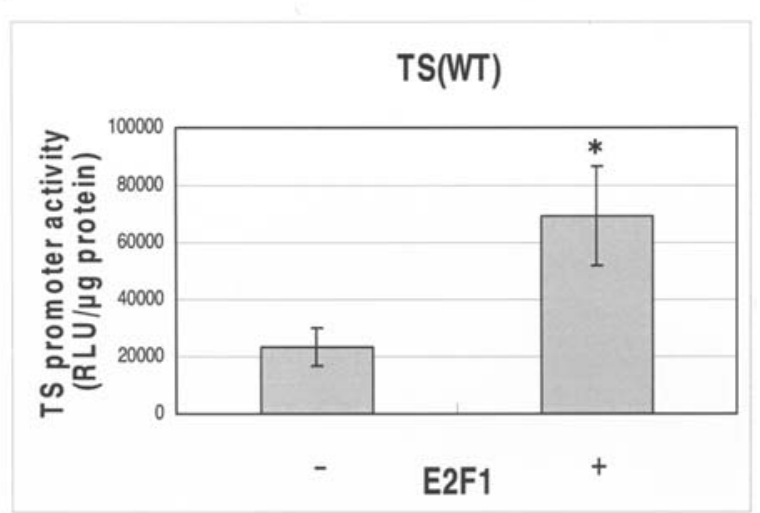

C

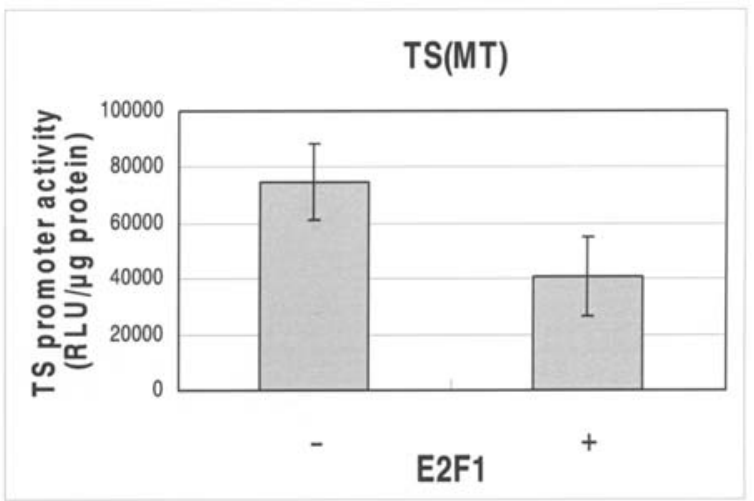

Figure 3. (A) Effect of ectopic p21 expression on TS promoter activity. p21 expression vector was transfected with TS reporter plasmid at the same time, and a luciferase assay was performed $\left({ }^{*} \mathrm{p}<0.05\right)$. (B) Effect of ectopic E2F1 expression on TS promoter activity. E2F1 expression vector was transfected with TS reporter plasmid at the same time, and a luciferase assay was performed ( $\mathrm{p}<\mathrm{0} 0.05)$. (C) Effect of ectopic E2F1 expression on mutant TS promoter activity. E2F1 expression vector was transfected with the mutant TS reporter plasmid at the same time, and luciferase assay was performed.

CDK inhibitor down-regulates TS expression in DLD-1 cells. To investigate whether the inhibition of CDK contributes to the down-regulation of TS in DLD-1 cells, we examined the expression of TS protein and mRNA after CDKI treatment. As shown in Fig. 4A, we found by Western blotting that SU9516 (a CDKI) decreased TS protein expression in a
A

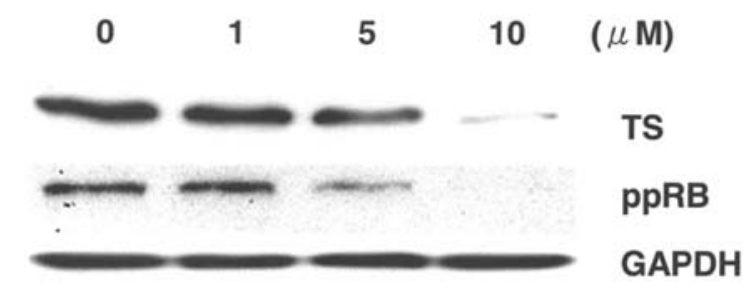

B

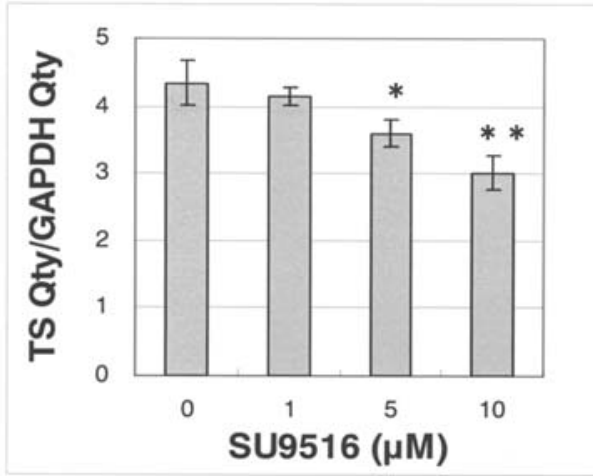

C

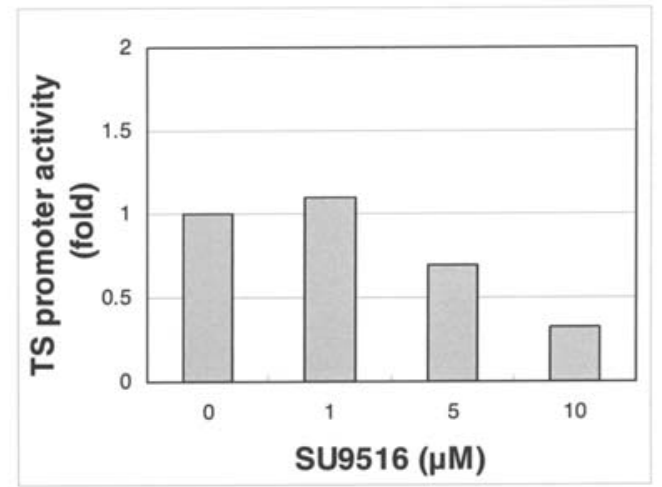

Figure 4. The effect of SU9516 on TS expression and RB status of DLD-1 cells. (A) DLD-1 cells were treated with SU9516 at the indicated concentrations for $24 \mathrm{~h}$. Western blotting was then performed. GAPDH was used as an internal control. (B) DLD-1 cells were treated with SU9516 at the indicated concentrations for $24 \mathrm{~h}$. Quantitative real-time RT-PCR was performed. The expression level of TS mRNA was normalized by the level of GAPDH mRNA in the same sample $\left({ }^{*} \mathrm{p}<0.05 ;{ }^{* *} \mathrm{p}<0.01\right)$. (C) DLD- 1 cells were transfected with the TS reporter plasmid and then treated with SU9516 at the indicated concentrations for $24 \mathrm{~h}$. The luciferase assay was then performed. Fold induction by SU9516 was calculated relative to that of DMSO (0).

dose-dependent manner, accompanied by the reduction of phosphorylated RB. In addition, we demonstrated, using quantitative real-time RT-PCR, that SU9516 also decreased TS mRNA expression in a dose-dependent manner (Fig. 4B). To examine whether the down-regulation of TS expression by CDKI is mediated through the TS promoter, we performed 
A

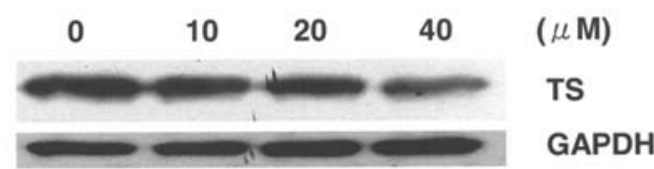

B

DLD-1 DLD-1/FdUrd

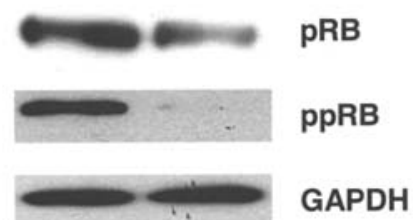

Figure 5. (A) The effect of SU9516 on TS expression of DLD-1/FdUrd cells. DLD-1/FdUrd cells were treated with SU9516 at the indicated concentrations for $24 \mathrm{~h}$. Western blotting was then performed. GAPDH was used as an internal control. (B) RB and the phosphorylated RB status of DLD-1 and DLD/FdUrd cells.

luciferase reporter assays using transient transfection of a TS promoter-luciferase reporter plasmid. SU9516 treatment of DLD-1 cells reduced the luciferase activity of the TS promoter in a dose-dependent manner (Fig. 4C). These results suggest that the CDKI decreases the TS promoter activity, mRNA and protein expression through the RB pathway in DLD-1 cells.

CDK inhibitor does not down-regulate TS expression in DLD-1/FdUrd cells similarly as in DLD-1 cells. To assess whether the same phenomena occur in DLD-1/FdUrd cells, we examined the expression of TS protein after CDKI treatment to DLD-1/FdUrd cells. As shown in Fig. 5A, the CDK inhibitor did not down-regulate TS expression in DLD-1/ FdUrd cells by the same concentrations as in DLD-1 cells . Upon confirming the RB status in DLD-1 and DLD-1/FdUrd cells by Western blotting, we found that expression of $\mathrm{RB}$ was quite low in DLD-1/FdUrd cells (Fig. 5B). These findings suggest that down-regulation of TS by a CDKI is affected by the status of RB where the CDKI shows the effect.

CDK inhibitor up-regulates the sensitivity to FdUrd in DLD-1 cells. We investigated the effects of a CDKI on the sensitivity to FdUrd in DLD-1 cells. Fig. 6 shows the colony formation assay of DLD-1 cells in the presence of FdUrd after treatment with DMSO or SU9516. At concentrations of FdUrd between 0.1-0.5 $\mu \mathrm{M}$, the numbers of colonies of SU9516-treated cells were lower than those of DMSO-treated cells. This result indicates that the CDKI up-regulated the sensitivity of DLD-1 cells to FdUrd.

\section{Discussion}

As already performed in 5-FU-mediated chemotherapy, one method of improving the response rate is the combined use of 5-FU with an inhibitor of dihydropyrimidine dehydrogenase, which degrades 5-FU (22). Nevertheless, the response rate is not exceptionally high because the expression levels of TS are elevated in many cases causing drug resistance (3). In the present study, in an attempt to identify a new chemo-

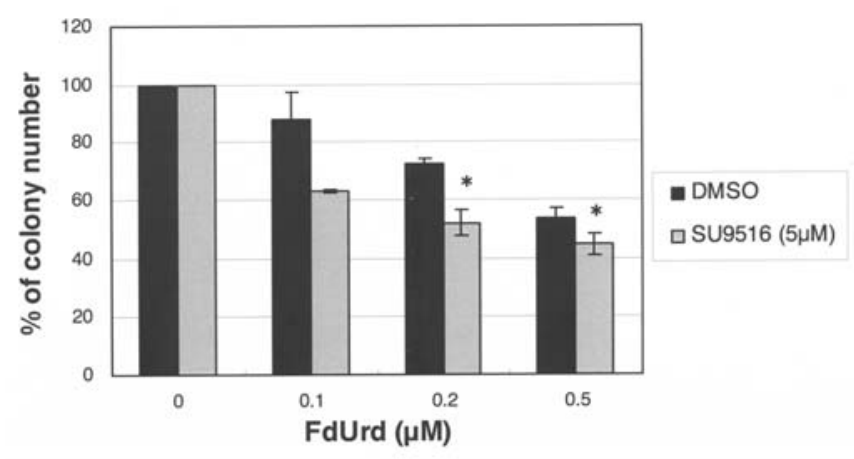

Figure 6. Effect of SU9516 on the sensitivity of DLD-1 cells to FdUrd. Cells were treated with DMSO or SU9516 $(5 \mu \mathrm{M})$ for $24 \mathrm{~h}$ and then exposed to various concentrations of FdUrd for $48 \mathrm{~h}$, and the number of viable colonies was counted $\left({ }^{*} \mathrm{p}<0.05\right)$

sensitizer, we used a CDKI as a suppressor of TS. As expected, we found that the treatment of DLD-1 cells with SU9516 reduced TS expression, resulting in enhancement of the sensitivity to FdUrd. CDKIs, by themselves are attractive compounds for molecular-targeting chemotherapy. From our experiments, CDKIs are expected to be not only attractive compounds for molecular-targeting chemotherapy, but also attractive chemosensitizers to 5-FU. However, the effect of SU9516 as a suppressor of TS might be reduced by low RB expression, which was observed in DLD-1/FdUrd cells. This indicates that other regimens must be utilized in the case that $\mathrm{RB}$ expression is low, as observed in DLD-1/FdUrd cells which were established by the long exposure to FdUrd (16).

Our promoter analysis of TS suggested that the CDKI acts as a counter agent against E2F. The negative regulation of cellular proliferation by $\mathrm{RB}$ is attributed to its transcriptional suppression of E2F target genes (10). The results of the present study indicate that down-regulation of TS expression by SU9516 is at least partially mediated through the status of RB phosphorylation.

In conclusion, we showed that a CDKI reduced TS expression and enhanced the sensitivity to FdUrd in DLD-1 cells. The results indicate the possibility that addition of a CDKI to 5-FU-mediated chemotherapy might be a good method for molecular-targeting chemotherapy against colorectal cancers. Furthermore, we expect that inducers of CDKIs or activators of RB might also be useful compounds in combination with 5-FU-mediated chemotherapy.

\section{Acknowlegements}

We thank Drs Y. Sugimoto, H. Tsujimoto and M. Fukushima (Taiho Pharmaceutical Co. Ltd.) for the generous gifts. This work was supported in part by a Grant-in-Aid for Scientific Research from the Ministry of Education, Culture, Sports, Science and Technology of Japan and a Grant-in-Aid for Scientific Research from the Japan Society for the Promotion of Science.

\section{References}

1. Pinedo HM and Peters GF: Fluorouracil: biochemistry and pharmacology. J Clin Oncol 6: 1653-1664, 1988. 
2. Rahman L, Voeller D, Rahman M, Lipkowitz S, Allegra C, Barrett JC, Kaye FJ and Zajac-Kaye M: Thymidylate synthase as an oncogene: a novel role for an essential DNA synthesis enzyme. Cancer Cell 5: 341-351, 2004.

3. Peters GJ, Backus HH, Freemantle S, van Triest B, Codacci-Pisanelli G, van der Wilt CL, Smid K, Lunec J, Calvert AH, Marsh S, McLeod HL, Bloemena E, Meijer S, Jansen G, van Groeningen CJ and Pinedo HM: Induction of thymidylate synthase as a 5-fluorouracil resistance mechanism. Biochim Biophys Acta 1587: 194-205, 2002.

4. Aschele C, Lonardi S and Monfardini S: Thymidylate synthase expression as a predictor of clinical response to fluoropyrimidine-based chemotherapy in advanced colorectal cancer. Cancer Treat Rev 28: 27-47, 2002.

5. Welsh SJ, Titley J, Brunton L, Valenti M, Monaghan P, Jackman AL and Aherne GW: Comparison of thymidylate synthase (TS) protein up-regulation after exposure to TS inhibitors in normal and tumor cell lines and tissues. Clin Cancer Res 6: 2538-2546, 2000.

6. Schmitz JC, Chen T and Chu E: Small interfering doublestranded RNAs as therapeutic molecules to restore chemosensitivity to thymidylate synthase inhibitor compounds. Cancer Res 64: 1431-1435, 2004.

7. Yang Z, Cloud A, Hughes D and Johnson L: Stable inhibition of human thymidylate synthase expression following retroviral introduction of an siRNA gene. Cancer Gene Ther 13: 1-8, 2006.

8. Degregori J, Kowalik T and Nevins JR: Cellular targets for activation by the E2F1 transcription factor include DNA synthesis- and G1/S-regulatory genes. Mol Cell Biol 15: 4215-4224, 1995.

9. Angus SP, Wheeler LJ, Ranmal SA, Zhang X, Markey MP, Mathews CK and Knudsen ES: Retinoblastoma tumor suppressor targets dNTP metabolism to regulate DNA replication. J Biol Chem 277: 44376-44384, 2002.

10. Mayhew C, Perkin L, Zhang X, Sage J, Jacks T and Knudsen ES: Discrete signaling pathways participate in RB-dependent responses to chemotherapeutic agents. Oncogene 23: 4107-4120, 2004.

11. Dyson N: The regulation of E2F by pRB-family proteins. Genes Dev 12: 2245-2262, 1998 .

12. Banerjee D, Gorlick R, Liefshitz A, Danenberg K, Danenberg PC, Danenberg PV, Klimstra D, Jhanwar S, Cordon-Cardo C, Fong Y, Kemeny N and Bertino JR: Levels of E2F-1 expression are higher in lung metastasis of colon cancer as compared with hepatic metastasis and correlate with levels of thymidylate synthase. Cancer Res 60: 2365-2367, 2000.
13. Kasahara M, Takahashi Y, Nagata T, Asai S, Eguchi T, Ishii Y, Fujii $M$ and Ishikawa K: Thymidylate synthase expression correlates closely with E2F1 expression in colon cancer. Clin Cancer Res 6: 2707-2711, 2000.

14. Lane ME, Yu B, Rice A, Lipson KE, Liang C, Sun L, Tang C, McMahon G, Pestell RG and Wadler S: A novel cdk2-selective inhibitor, SU9516, induces apoptosis in colon carcinoma cells. Cancer Res 61: 6170-6177, 2001.

15. Moshinsky DJ, Bellamacina CR, Boisvert DC, Huang P, Hui T, Jancarik J, Kim SH and Rice AG: SU9516: biochemical analysis of CDK inhibition and crystal structure in complex with cdk2. Biochem Biophys Res Commun 310: 1026-1031, 2003.

16. Murakami Y, Kazuno H, Emura T, Tsujimoto H, Suzuki N and Fukushima M: Different mechanism of acquired resistance to fluorinated pyrimidines in human colorectal cancer cells. Int $\mathbf{J}$ Oncol 17: 277-283, 2000.

17. Waldman T, Kinzler K and Vogelstein B: p21 is necessary for the p53-mediated G, arrest in human cancer cells. Cancer Res 55: 5187-5190, 1995.

18. Okabe H, Tsujimoto $\mathrm{H}$ and Fukushima M: Preparation of the antibodies against recombinant human thymidylate synthase for the detection of its intratumoral levels and the application to sensitivity-study of 5-fluorouracil. Oncol Rep 4: 685-690, 1997.

19. Fujiwara H, Terashima M, Irinoda T, Fujiwara H, Terashima M, Irinoda T, Takagane A, Abe K, Kashiwaba M, Oyama K, Takahashi M, Maesawa C, Saito K, Takechi T and Fukushima M: Quantitative measurement of thymidylate synthase and dihydropyrimidine dehydrogenase mRNA level in gastric cancer by real-time RT-PCR. Jpn J Cancer Res 93: 1342-1350, 2002.

20. Kawakami K, Salonga D, Park JM, Danenberg KD, Uetake H, Brabender J, Omura K, Watanabe G and Danenberg PV: Different lengths of a polymorphic repeat sequence in the thymidylate synthase gene affect translational efficiency but not its gene expression. Clin Cancer Res 7: 4096-4101, 2001.

21. Obama K, Kanai M, Kawai Y, Fukushima M and Takabayashi A: Role of retinoblastoma protein and E2F-1 transcription factor in the acquisition of 5-fluorouracil resistance by colon cancer cells. Int J Oncol 21: 309-314, 2002.

22. Hoff PM: The tegafur-based dihydropyrimidine dehydrogenase inhibitory fluoropyrimidines, UFT/leucovorin (ORZEL) and S-1: a review of their clinical development and therapeutic potential. Invest New Drugs 18: 331-342, 2000. 